\title{
Clinical review: peripheral muscular ultrasound in the ICU
}

\author{
Paolo Formenti ${ }^{1,3^{*}}$, Michele Umbrello ${ }^{1,3}$, Silvia Coppola ${ }^{1,3}$, Sara Froio ${ }^{1,3}$ and Davide Chiumello $o^{1,2,3}$
}

\begin{abstract}
Muscular weakness developing from critical illness neuropathy, myopathy and muscle atrophy has been characterized as intensive care unit-acquired weakness (ICUAW). This entity occurs commonly during and after critical care stay. Various causal factors for functional incapacity have been proposed. Among these, individual patient characteristics (such as age, comorbidities and nutritional status), acting in association with sustained bed rest and pharmacological interventions (included the metabolic support approach), seem influential in reducing muscular mass. Long-term outcomes in heterogeneous ICUAW populations include transient disability in 30\% of patients and persistent disabilities that may occur even in patients with nearly complete functional recovery. Currently available tools for the assessment of skeletal muscle mass are imprecise and difficult to perform in the ICU setting. A valid alternative to these imaging modalities is muscular ultrasonography, which allows visualization and classification of muscle characteristics by cross-sectional area, muscle layer thickness, echointensity by grayscale and the pennation angle). The aim of this narrative review is to describe the current literature addressing muscular ultrasound for the detection of muscle weakness and its potential impact on treatment and prognosis of critically ill patients when combined with biomarkers of muscle catabolism/anabolism and bioenergetic state. In addition, we suggest a practical flowchart for establishing an early diagnosis.
\end{abstract}

Keywords: ICU-acquired weakness, Skeletal muscle, Peripheral muscular ultrasound, Muscle cross-sectional area, Muscle layer thickness, Pennation angle, Muscle echointensity

\section{Background}

Many patients admitted to the intensive care unit (ICU) develop a syndrome of neuromuscular dysfunction characterized by generalized muscle weakness that often contributes to difficult liberation from mechanical ventilation [1]. The principal causes of such weakness include neuropathic and myopathic disorders, as well as mixed disorders that have been lumped under the term "ICUacquired weakness" (ICUAW) [2]. Since this syndrome occurs in the absence of pre-existing neuromuscular disease and affects all age groups [3-6], ICUAW is believed to reflect illnesses or treatments occurring in the ICU [7]. Recent studies demonstrated that a reduced excitability of the nerve and muscle cell membranes might contribute

*Correspondence: formenti.paolo80@gmail.com

1 SC Anestesia e Rianimazione, Ospedale San Paolo - Polo Universitario, ASST Santi Paolo e Carlo, Milan, Italy

Full list of author information is available at the end of the article to the development of ICUAW during the acute stages of the polyneuropathy and myopathy encountered in critically ill patients [8-11]. This association has been linked with severe systemic responses to infection, as well as with mortality rates of $30-50 \%$ and transient disability in $30 \%$ of the cases [12]. Persisting disabilities often linger in patients with apparently complete functional recovery [13]. Moreover, ICUAW is now recognized to be a very important factor in "difficult-to-wean" patients in the ICU setting, associated with prolonged ICU/hospital stays $[14,15]$. Regarding the development of ICUAW, many factors play a key role, even if the pathogenesis of this syndrome is far from being completely understood $[2,16]$. Among these, individual patient features, such as age and comorbidities [17, 18], sustained bed rest [19], pharmacological strategies, nutritional and metabolic support, all seem to be related with the reduction in muscle mass [20]. In order to improve nutritional and rehabilitation strategies, different diagnostic methods to 
diagnose ICUAW at an early stage have been investigated [21-23]. The currently available tools for the assessment of skeletal muscle mass with the highest level of accuracy and reproducibility are computerized tomography (CT) and magnetic resonance imaging (MRI); however, high-precision imaging is difficult to perform in the ICU setting. On the contrary, high-resolution ultrasound machines that are now routinely available represent valid and reliable tools for providing qualitative and quantitative details about muscle disease [24-28]. Few systematic reviews have been focused on this topic [29, 30], and the authors did not perform formal meta-analyses due to issues with the design of the studies included, the heterogeneity of patient cohorts, and the different aspects of peripheral skeletal muscle architecture measured. Since then, few papers have focused on the validation of methodologies [28, 31] and others investigated different muscle zones and ultrasound characteristics [32-35]. Ultrasound is able to derive structural variables, and, once it is performed repeatedly, it is able to identify those patients at highest risk of prolonged complications, which result from excess muscle catabolism. Thus, the aim of this narrative review is to describe the current literature about the use of muscular ultrasound for detecting muscle weakness and its impact on critically ill patients' treatments and prognosis. Moreover, we propose a flowchart with the aim of using muscular ultrasound together with clinical examination for an early diagnosis of pathology and etiology.

\section{Ultrasound assessment of muscular features}

As a noninvasive, painless technique, ultrasound may be used to identify skeletal muscle pathology. It offers several advantages compared with other tests used in the evaluation of muscle features and allows for a quick screen of large muscle areas at the bedside. In fact, healthy muscle tissue has a distinctive appearance on ultrasound that readily distinguishes it from other tissues (Fig. 1) [36, 37]. To perform an adequate ultrasound examination of skeletal muscle, several technical components must be considered. First, since muscle and subcutaneous fat can easily be compressed, a minimal amount of pressure should be applied on the tissue under an ultrasound probe sufficiently covered with gel, in order to optimize imaging conditions. Additionally, obesity and subcutaneous edema can significantly alter the appearance and quality of the ultrasound images of skeletal muscle. Therefore, the examiner must be aware

\section{1- Healthy subject}

\section{2- Long term ICU patients}
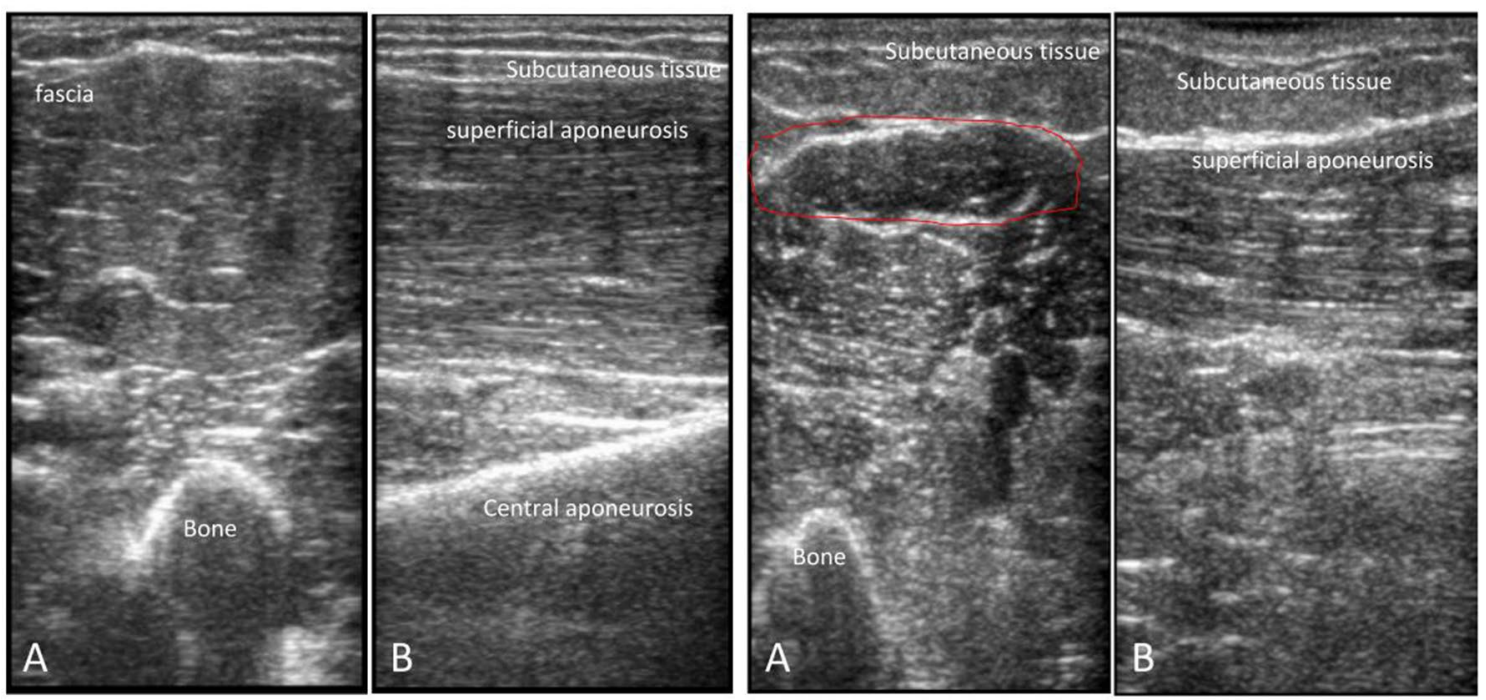

Fig. 1 Ultrasound distinctive appearance of muscle tissue. The figure shows a transverse (a) and longitudinal (b) ultrasound scan of elbow flexor (bicep brachialis) in healthy (1) and long-term ICU (2) subjects. In the axial image, muscle consists of primarily hyper-echogenic areas scattered with small bright curved echoes of superficially random orientations. In the sagittal plane, these bright echoes are seen to be the fibrous tissue that surrounds muscle fibers and fascicles and which organize into recognizable striations. In bipennate or multi-pennate muscles, a central aponeurosis can be identified as an area of thickened fibrous tissue that when followed distally becomes the tendon. Bone is highly echogenic with a deep shadow beneath the bright hard edge. Subcutaneous fat is typically of similar echogenicity to muscle and is interposed with brighter, poorly organized strips of connective tissue. Near the myotendinous junction, the myofascial fibrils merge, resulting in increased echogenicity and higher anisotropy. In the healthy tissue, the hyper-echogenic muscle is interspersed with bright fibro adipose tissue and the bone reflection is bright and sharply defined; in the long-term ICU patient, the muscle tissue appears as non-homogenous and reduced in its mass 
of the depth of the imaged tissue, the potential effects of attenuation of the ultrasound signal, and the limitations of the ultrasound system in use, taking into account the gain, the focal points and the compression, as all of these factors may significantly alter the overall appearance of myofascial structures [24]. The probe orientation and muscle position can also radically alter the image appearance, given that the relationship between the probe angle and the underlying pennation angle of the myofascial strips critically modulates the ultrasonographic brightness of the muscle [38]. The most important factors that contribute to the heterogeneity in muscle detection are the transducer selection frequency and the field of view. Using a linear probe, the frequencies for the clinical evaluation of neuromuscular measures range from approximately 2-20 MHz. Radiologic imaging is increasingly used as a diagnostic tool to describe the patterns and extent of muscle involvement, thanks to modern techniques that enable determinations of muscle atrophy and changes in connective tissue. Such methodologies usually grade the severity of the disease process with greater accuracy than clinical scores. CT has made possible the evaluation and selective involvement of muscle groups, establishing disease progression and identifying asymptomatic involvement [39]. CT scan is usually more accessible than MRI. Moreover, it allows for quick and accurate evaluation of muscle changes, especially fatty degeneration, by assessing muscle density and morphology. Such examinations are not operator dependent and allow for the evaluation of the deepest muscles. However, CT scanning involves high doses of ionizing radiation. MRI provides excellent soft tissue characterization, so it perfectly evaluates shape, volume and morphological features of normal skeletal muscle allowing for determination of fatty degeneration in late-stage muscle dystrophy, aside from recognition of decreases in muscle volume [40]. Unfortunately, MRI incurs high cost and lacks portability. Cumbersome study logistics precludes its routine clinical use in the ICU setting. Nerve and muscle biopsies are invasive and expensive, hold potential for complications, and require specialized expertise for obtaining and interpreting samples. Although the classical diagnostic tool to detect ICUAW is the combination of nerve conduction and electromyographic studies (EMG), there are many technical challenges to completing both in the ICU [41]. Commonly encountered physiologic changes in the ICU, such as anasarca and hypothermia, introduce artifacts by altering amplitude and velocity recordings for nerve conduction studies [42]. Finally, the utility of manual muscle strength assessment using standardized scales such as that of the Medical Research Council proves useful in patients who are both sufficiently awake and cooperative [43]. A recent study investigated the accuracy of muscular and nerve ultrasound for the diagnosis of ICUAW compared with this benchmark test [44]. That study showed the MRC to have very low diagnostic accuracy for all muscle and nerve parameters. Considering the limitations of traditional methods, together with recent advances in muscular ultrasound, makes ultrasonography a promising tool for the study of muscle structure, facilitating early diagnosis and intervention.

\section{Parameters of muscle architecture Cross-sectional area}

The cross-sectional area (CSA) is determined by the number and size of individual fibers within a muscle. It is comprised of two areas: anatomical (cross section of a muscle perpendicular to its longitudinal axis) and physiological (cross section of a muscle perpendicular to its fibers, generally at its largest diameter). The term 'muscle architecture' (parallel or pennate) refers to the physical arrangement of muscle fibers at the macroscopic level and determines the muscle's mechanical function. In a parallel muscle, the two CSAs coincide, as the fibers are parallel to the longitudinal axis. In pennate muscles, both areas may be used to describe the contraction properties (Fig. 2). In fact, since muscle strength relates to muscle volume, the latter may be inferred from its CSA [30]. Because these measurements do not need muscle tension, they are often assessed instead of muscle strength tests [45], especially in non-cooperative patients. Muscle atrophy mainly affects fast fibers (type II) rather than a relatively equal loss of slow and fast fibers. This loss potentially results in a drop in physical activity levels, in the denervation/re-innervation process, and in reduced synthetic rates of muscle proteins. Thus, muscle bulk can be measured by the CSA, whose variation is dependent on age, gender, and muscle group [46].

\section{Muscle layer thickness}

Muscle thickness, the distance between two fasciae, is easily identifiable with ultrasound (Fig. 3). Its reliability has been previously reported in comparison with other imaging modalities as well as direct measurements on dissected cadavers [25, 47, 48], while its reproducibility has been defined as the highest in various muscles $[26,49,50]$. Since CSA is directly related to the loss of strength, some authors tried to predict CSA directly from muscle thickness. Although the two parameters significantly correlate [51], the prediction of CSA from muscles thickness has not been proven [26]. Thus, even if it has been shown that muscle loss of ICU patients could be monitored by thickness measurements [52], we suggest that other indexes reflecting muscle strength should be added to muscle thickness in order to improve precision. 


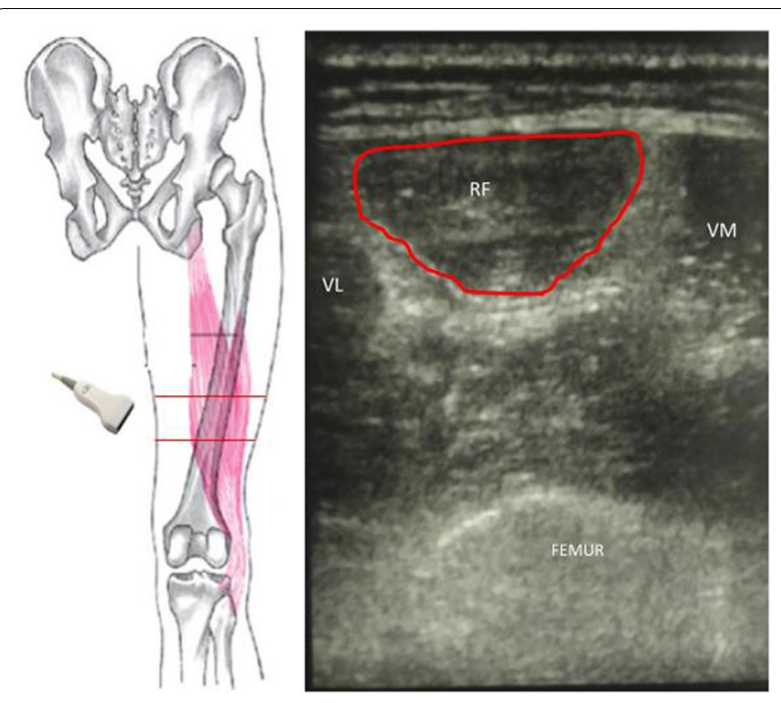

Fig. 2 Muscle cross-sectional area. This figure depicts the cross-sectional area of the rectus femoris perpendicular to its longitudinal axis. The quadriceps femoris is a group of muscles composed by three vastus muscles (medialis, intermedius, and lateralis) and the rectus femoris which presents a proximal insertion in the anterior inferior iliac spine and other insertion in the supra-acetabular sulcus. Left side: standardized level of ultrasound scan of the lower limb; in the supine position, the probe should be placed at 2/5 of an imaginary line between the anterior parts of the thigh from the anterior inferior iliac spine to the midpoint of the proximal border of the patella. Right side: the figure depicts the cross-sectional area (red circle) of the rectus femoris (RF) perpendicular to its longitudinal axis. VI vastus intermedius, VM vastus medialis, $V L$ vastus lateralis

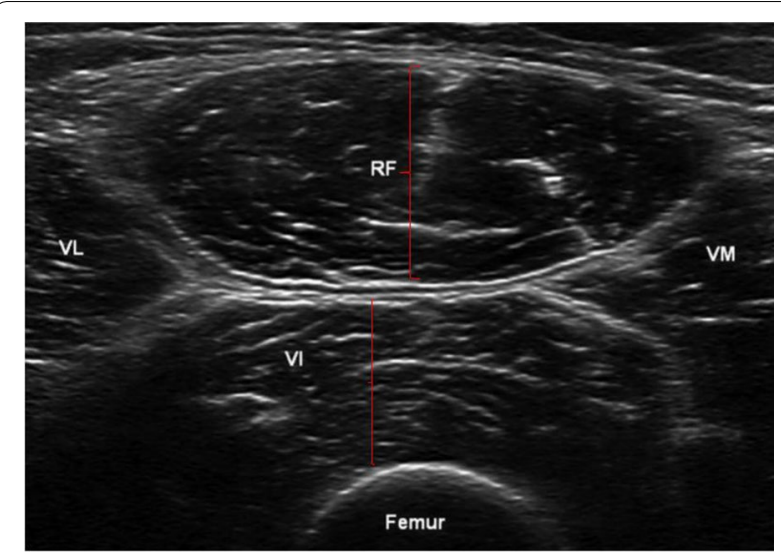

Fig. 3 The muscle layer thickness detected by ultrasound. Quadriceps femoris detected by ultrasound in a transverse scan. The rectus femoris (RF) layer thickness and vastus intermedius (VI) are represented (red lines). VM vastus medialis, VL vastus lateralis

\section{Echointensity}

Information about muscle composition can be gathered by quantification of muscle echogenicity [53]. The measure of the image grayscale reflect the muscle's composition: increased echogenicity indicates more homogenous muscle [54]. Echointensity is calculated by performing grayscale analysis of image pixels. Briefly, all the pixels in a selected area of the muscle are categorized on a grayscale configuration using a standard histogram function widely available in many commercially available types of software for image editing (Fig. 4). Quantitative grayscale analysis has proven to be better than visual assessment alone of ultrasound images [37], but it is slightly more time consuming and requires the establishment of normal reference values. Ultrasonic echogenicity can be graded according to a score that classifies ultrasonic echogenicity semiquantitatively into four levels, with higher grades corresponding to increased severity of muscle impairment [55]. Graded echogenicity has been shown to correlate with muscle pathologic findings on biopsy [53]. As with other measures, echogenicity measurements are highly influenced by observer-dependent factors, such as the adjustment of the ultrasound probe. Additional, factors, such as hydration balance, might also have an impact.

\section{Pennation angle}

As mentioned above, muscle architecture can be described by the pennation angle, i.e., the angle of insertion of muscle fibers into the aponeurosis (Fig. 5). This angle provides information about muscle strength, as the greater the pennation angle, the more the contractile material packed within a given volume and by inference, the higher is the muscle's capacity to generate force [56]. Moreover, pennation angle has been shown to be significantly correlated with the CSA [57]. Therefore, the angle of pennation is critical for determining force dynamics of muscle. Because pennation angle measurements are strongly influenced by adjustment of the ultrasound probe, some authors have expressed concerns regarding the observer dependency of this technique [58]. In particular, its reproducibility in muscles other than the quadriceps has been reported low [38]. Eventually, the fascicle length (FL) can be derived from pennation angle and muscle thickness, as described elsewhere [51] using the following formula: $\mathrm{FL}=\mathrm{TH} /(\sin \mathrm{PA})$, where $\mathrm{TH}$ is muscle thickness and PA the pennation angle. Muscles with larger pennation angles are thicker, as they have greater numbers of sarcomeres in parallel with the direction of the fascicle. It is possible that these parallel sarcomeres are lost first, causing loss of pennation angle as an indication of reduced thickness.

\section{Muscular ultrasound in clinical practice}

Lower limbs muscles are more subject to early atrophy than those of the upper limbs [32]. The quadriceps, the largest muscle group of the lower limb, is the one 

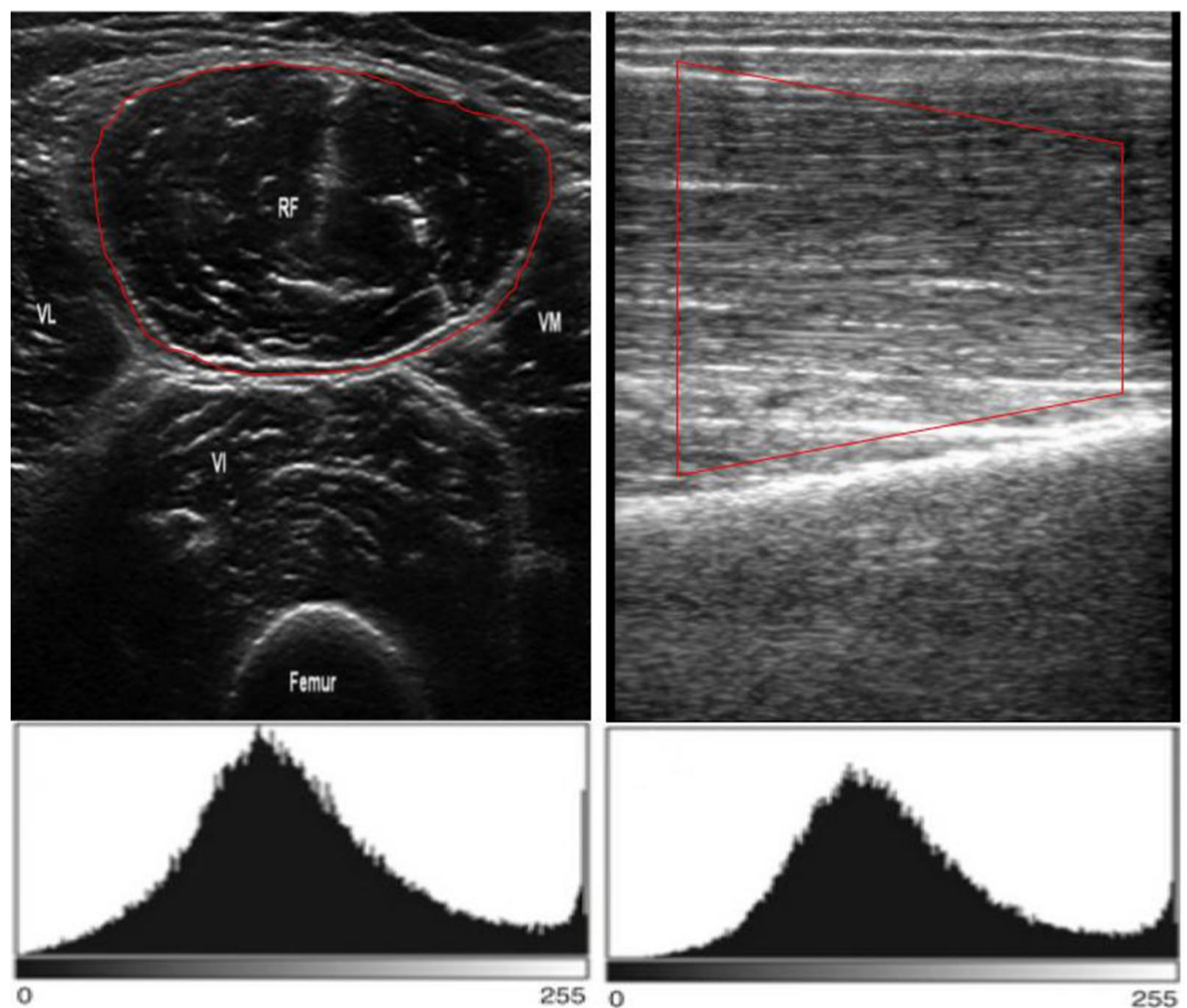

Fig. 4 The muscle ultrasound echointensity. An example of the grayscale histogram in the transverse (right) and longitudinal (left) axis of the rectus femoris
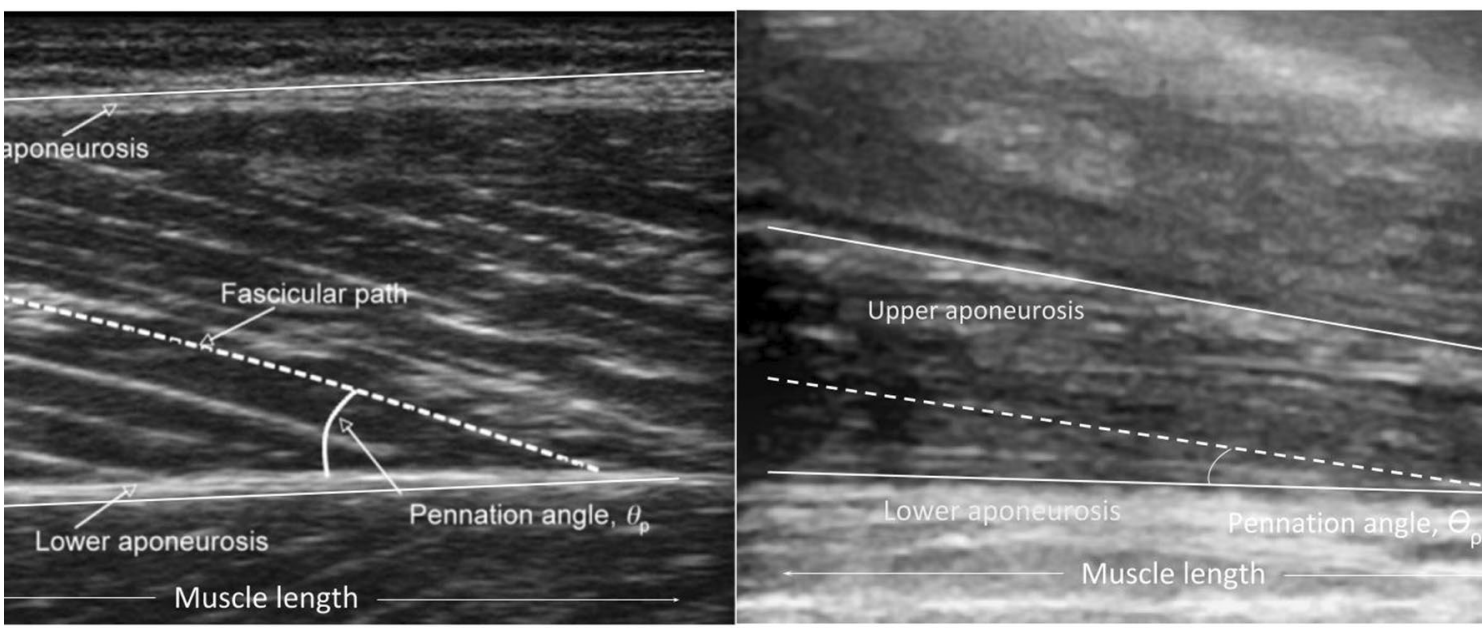

Fig. 5 The muscle ultrasound pennation angle. The figure represents a longitudinal view of quadriceps rectus femoris muscle. The pennation angle is calculated between the intercept of fascicular path to the lower aponeurosis. Additionally, the muscle length can be measured. These two variables may be used to determine the strength of the muscle, as the lower is the angle, the lower is the length, and the lower is the strength. The right panel represents a representative reduction in pennation angle after 1 week of ICU stay 
generally explored with ultrasound. The image obtained allows assessments of muscle thickness, area, and ultrasound pattern, information, which can monitor contraction patterns that characterize muscular physiology and pathology. In the following section, we will describe the evidence on the use of this technique in critically ill patients (Table 1). Our comprehensive bibliographic search strategy accessed the following databases: PubMed, CINAHL, Cochrane Library, Scopus, Web of Science, from their inception to the cutoff date of July 31, 2018. The following keywords were used, alone or combined with appropriate Boolean operators, to search these databases: "muscular," "peripheral muscular," "ultrasound," "intensive care unit," "critical care," "critical illness," "weakness."

\section{Muscular ultrasound in the ICU setting}

Many studies examining the association between muscle weakness and clinical outcome have reported how muscle weakness was an independent predictor of mortality [59], increased ventilator-dependent time [60] and prolonged ICU length of stay (LOS) [61]. In particular, a negative correlation has been shown between muscular thickness (in both upper and lower limbs) and ICU LOS [52, 62, 63]. Several studies that investigated alterations in anabolic and catabolic signaling have introduced the use of ultrasound for the detection of muscular characteristics with the aims to improve pathophysiological knowledge of strength and to aid early diagnosis. However, interpretation of such reports is difficult because of significant methodological variability (such as small sample sizes and the lack of standardization of imaging assessment). Moreover, we are currently unaware of how sex, age and presenting illness affect loss of muscle mass in the critically ill. In most studies reliability of the ultrasonic technique was not the primary targeted outcome, which helps explain the lack of methodological consistency and precision. Some studies reported assessments of quantitative ultrasound reliability for measures of muscle linear depth, only two for CSA, and only one for echogenicity. Puthucheary et al. [16] showed a correlation coefficient $\left(R^{2}\right)$ of 0.97 for measuring rectus femoris CSA between two blinded independent raters. Baldwin published two observational methodological studies evaluating the reliability of muscle linear depth of midupper arm, mid-forearm, and mid-thigh and reported intra-rater intra-class correlation coefficients ranging from 0.998-1.0 [64] and $\geq 0.976$ [65]. Regarding the muscle thickness, Gruther et al. [52] reported a coefficient of variation on repeated anterior thigh mean linear depth measures of $0.25 \%$, without assessing the intra- or inter-rater reliability. More recently, Hadda [31] showed an excellent intra-observer $(>0.997)$ and inter-observer
(0.963) agreement among five evaluators measuring arm muscle thickness. Finally, Grimm [66] also assessed muscle echogenicity, showing good inter-rater (0.915) and intra-rater (0.972) coefficients.

Another issue that should be taken into account is that most of the studies of ICUAW have been performed under the assumption that abnormalities found by the authors are ICU-acquired; no study attempted to control for prehospital muscle function or overall functional status as a predictor of ICUAW [67]. In fact, the so-called age-related frailty syndrome is characterized by a the loss of muscle mass that seems to be encountered in up to $80 \%$ of elderly ICU patients even in the absence of ICUAW diagnosis [68]. This condition constitutes an important predictor of long-term mortality [69] and morbidity [68], but the impact of pre-existing frailty on ICUAW is still unknown.

\section{Upper limb assessment}

Most published studies investigated the lower limb muscles for the reasons explained above. Relatively few studies have selected the upper arm as their principal zone of interest, and when doing so sometimes compared it with other regions. Among these, Reid [66] performed serial measurements of mid-upper arm thickness within the first $72 \mathrm{~h}$ of ICU stay, showing how it decreased in almost every one of the 50 patients enrolled, independently of positive or negative energy balance. With a similar purpose, Baldwing et al. [65] more recently performed serial measurements of the thickness of the anterior mid-upper arm, mid-forearm in 16 septic ICU patients compared with healthy subjects. As expected, septic patients were significantly weaker than control participants, with significant differences recorded in the thickness and thickness/free fatty mass (FFM) of all peripheral muscles. Such data suggest that by 2 weeks of ICU admission, muscles of different functionality may not be equally affected by a combination of insults that occur during critical illness. Finally, Turton et al. [32] investigated the elbow flexor compartment, the medial head of gastrocnemius and the vastus lateralis muscle at admission and after 10 days in 22 ICU mechanically ventilated patients. Interestingly, this study showed no changes to the size of the elbow flexor compartment, and loss of muscle mass occurred preferentially in the lower limb. These data help justify interrogating the lower limb, as it appears to be the peripheral muscle group predisposed to develop early disuse atrophy among in critically ill patients. Moreover, the Turton study was the first to investigate the role of pennation angle. Patients who had a larger pennation angle at the day of admission had greater percentage reductions of pennation angle as well as of muscle thickness. 


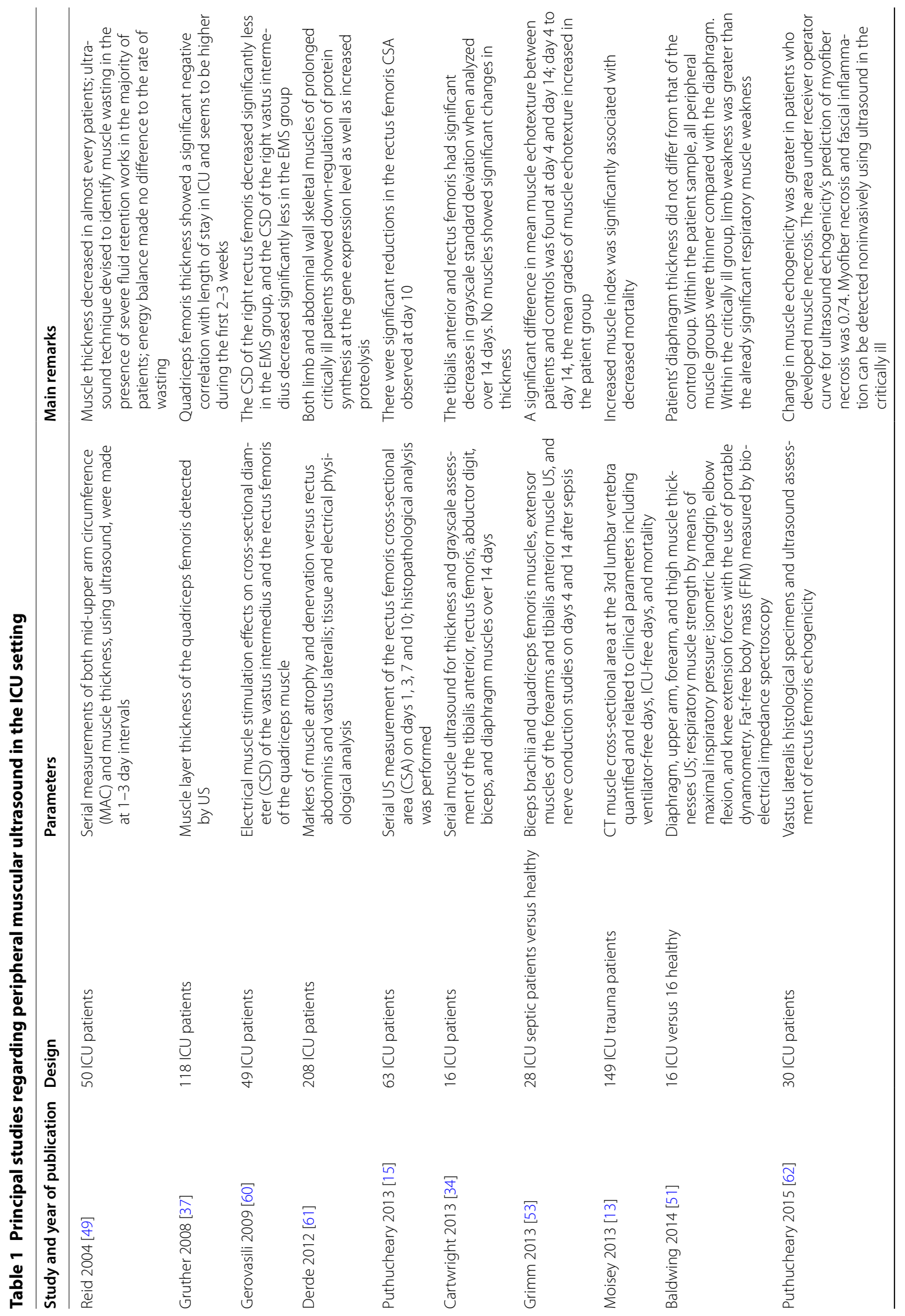




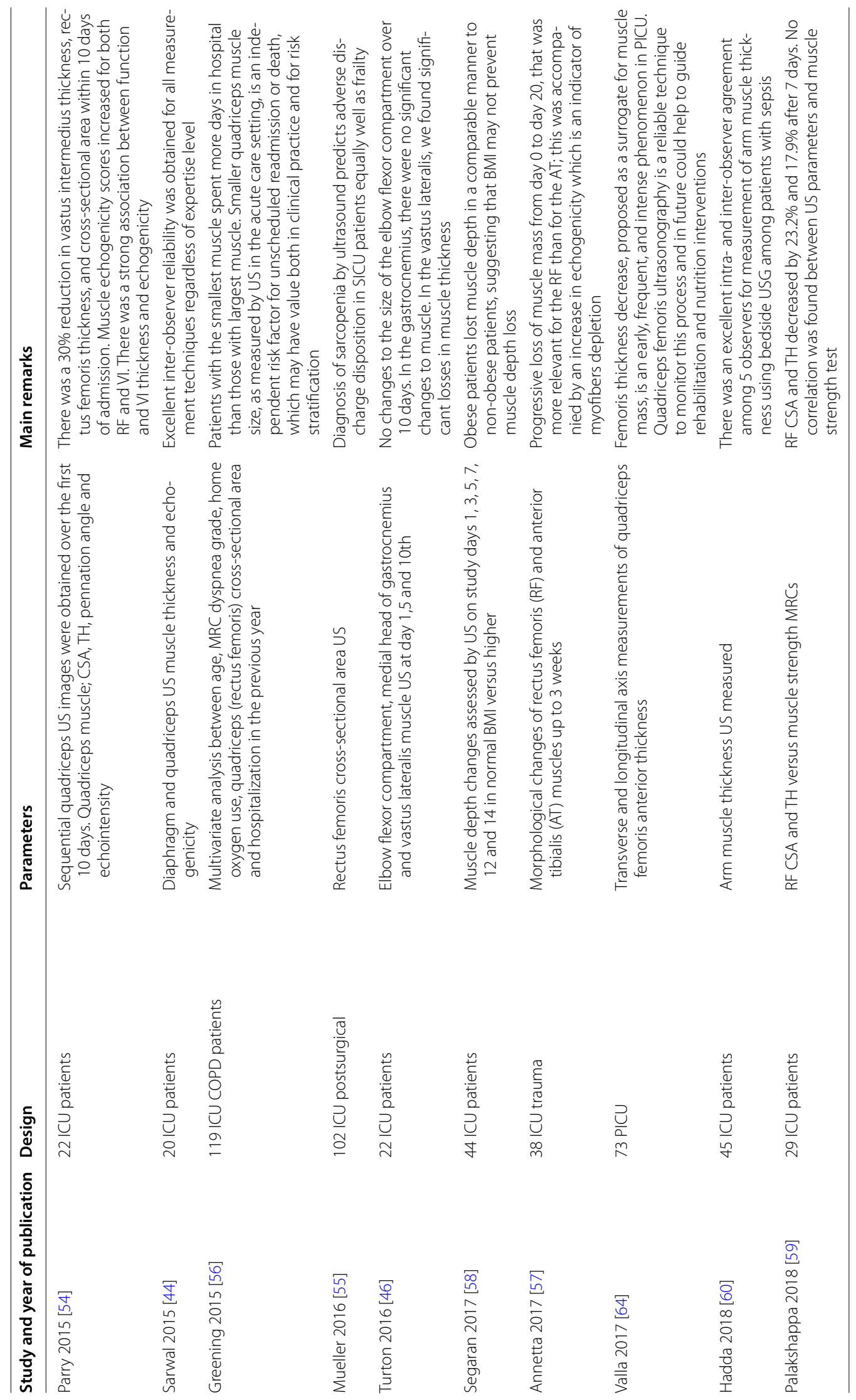




\section{Lower limb assessment}

Focusing largely on lower limb ultrasound investigations, most published studies considered the muscle layer thickness and the CSA parameters, whereas only a few papers assessed ultrasonic muscle echogenicity as the key parameter of interest. In this regard, Grimm et al. [66] found significant alterations in muscle echostructure in the early stage of sepsis compared with healthy controls. Since those patients were septic and had a positive fluid balance, it is difficult to clarify to what extent the observed change in muscle echogenicity was caused by edema rather than muscle wasting. However, as the authors pointed out, the significance of tissue edema in the assessment of muscle echogenicity may be overestimated, since tissue edema cannot alter the bone signal that is part of the echogenicity score. Moreover, since the muscle echostructure score increased during the first 2 weeks of care despite a concomitant decrease in fluid balance, a specific structural damage in muscle architecture has been assumed. Cartwright and colleagues [70] found similar observations in echostructure changes over 2 weeks in both the tibialis anterior and rectus femoris muscles. Interestingly, these changes were similar to those seen in other myopathic conditions and included a significant increase in mean grayscale value, indicating an increased muscle echogenicity, and a decrease in grayscale standard deviation, indicating that the muscle became more homogeneous [71]. The use of grayscale standard deviation to define muscle homogeneity is justified, considering that the standard deviation decreases as the pixels in the region of interest become more uniform. However, once again, it is difficult to define whether this pattern of change occurred because of muscle breakdown and loss of the normally well-organized muscle architecture or due to inflammation or fluid retention in the subcutaneous tissue and muscle. Since it is not clear if the ultrasonographic muscle changes correlate with strength, Parry et al. [72] addressed this topic and reported that muscle echogenicity scores increased in quadriceps muscle (both rectus femoris and intermedious vastus) by $12 \%$ and $25 \%$. Such observations suggest deterioration in muscle quality and establish a strong association between function and echogenicity. Eventually, in a recent prospective, two-center, observational study comparisons were made between sequential histological samples and ultrasound assessment of rectus femoris echogenicity [66]. This interesting paper showed how muscle echogenicity changes were greater in patients who developed muscle necrosis than in those who did not $(8.2 \%$ vs. $-15.0 \%)$. In a previous study [16], rectus femoris CSA and protein/DNA ratio were assessed over time, suggesting that all decreased over the first week. Hence, lower limb muscle wasting has suggested to occur as a consequence of both depressed muscle protein synthesis and an elevation in protein breakdown relative to protein synthesis, resulting in a net catabolic state. Unfortunately, muscle ultrasound significantly underestimated protein loss (as measured by the protein/DNA ratio), perhaps in part because of the presence of interstitial edema. Moving forward on CSA studies, there is only one paper that integrated the ultrasound values into a sarcopenia and frailty prediction model, showing how the rectus femoris CSA, adjusted for sex and integrated with nutrition, comorbidities, depression, and patient demographics data, was able to predict adverse discharge disposition in surgical ICU patients [33]. With similar purpose, looking at the risk of unscheduled readmission or death, Greening et al. [73] demonstrated how smaller quadriceps muscle size described by CSA in the acute care setting was an independent risk factor for subsequent unscheduled readmission. CSA has been also evaluated in selected critically ill populations - such as trauma and obesity-confirming the previous observations. In particular, a 3-week follow-up analysis of CSA and muscle diameter followed in ICU trauma patients showed how $100 \%$ of them experienced severe muscle mass loss. Approximately $45 \%$ of rectus femoris muscle mass was lost by day 20, together with a progressive increase in echogenicity score [34]. The muscle depth as a measure of muscle wasting was compared among obese, overweight and normal-weight patients using a muscle ultrasound technique [74]. Compared with a previous study that used a similar methodology, the muscle depth loss was comparable and not statistically different between the groups at each of the interrogated time points. Lastly, muscle thickness of different muscle groups was investigated in many studies, and the main results in the majority indicated that it was significantly reduced. Among these, as already mentioned, a $0.2-5.7 \%$ decrease/day has been described for the upper arm [66], and a similar percentage in the lower limb [36]. Interestingly, the progression of this reduction was not uniform among the different quadriceps muscles, with a $30 \%$ reduction in rectus femoris and vastus intermedius thickness and $14 \%$ reduction in vastus lateralis [56]. Palakshappa [35] described the relationship between rectus femoris CSA and quadriceps muscle thickness, with volitional measures of strength and function at 7 days after the admission in ICU in 29 patients with sepsis. The authors observed an expected decrease in both rectus femoris CSA and thickness (23.2\% and $17.9 \%$, respectively) but established only a moderate correlation with strength on day 7. Similarly, Puthucheary showed that thickness measurements significantly underestimate ICU muscle wasting compared with rectus femoris CSA [75]. 


\section{Practical issue}

Based on the current knowledge on this topic, we developed a methodological flowchart with the aim to diagnose ICUAW at an early stage and to optimize different patient-dependent factors, such as pharmacological strategies, muscular overloading or inactivity, and metabolic derangements (Fig. 6). Ideally, within the first $48 \mathrm{~h}$ after the admission to ICU, we suggest that a first muscular ultrasound assessment should be performed to paint a "baseline picture." We also suggest confining the evaluation to the quadriceps muscle, and in particular to the rectus femoris. At the same time, volitional strength evaluation, using validated tools such as the
Medical Research Council (MRC) scale [27, 43], should be performed as soon as cognitive impairment allows. The degree of possible cooperation should also be evaluated with validated scales for sedation, agitation level and delirium, such as the Richmond agitation sedation scale (RASS) and the confusion assessment method for the ICU (CAM-ICU) [76, 77]. In patients able to follow commands, manual muscle testing should be performed, with a score in the normal range confirming the absence of ICUAW. However, the necessary level of cooperation can reasonably be achieved on average only 8-10 days after ICU admission [72]. Impaired mental status or a low MRC score dictates the need for additional examination

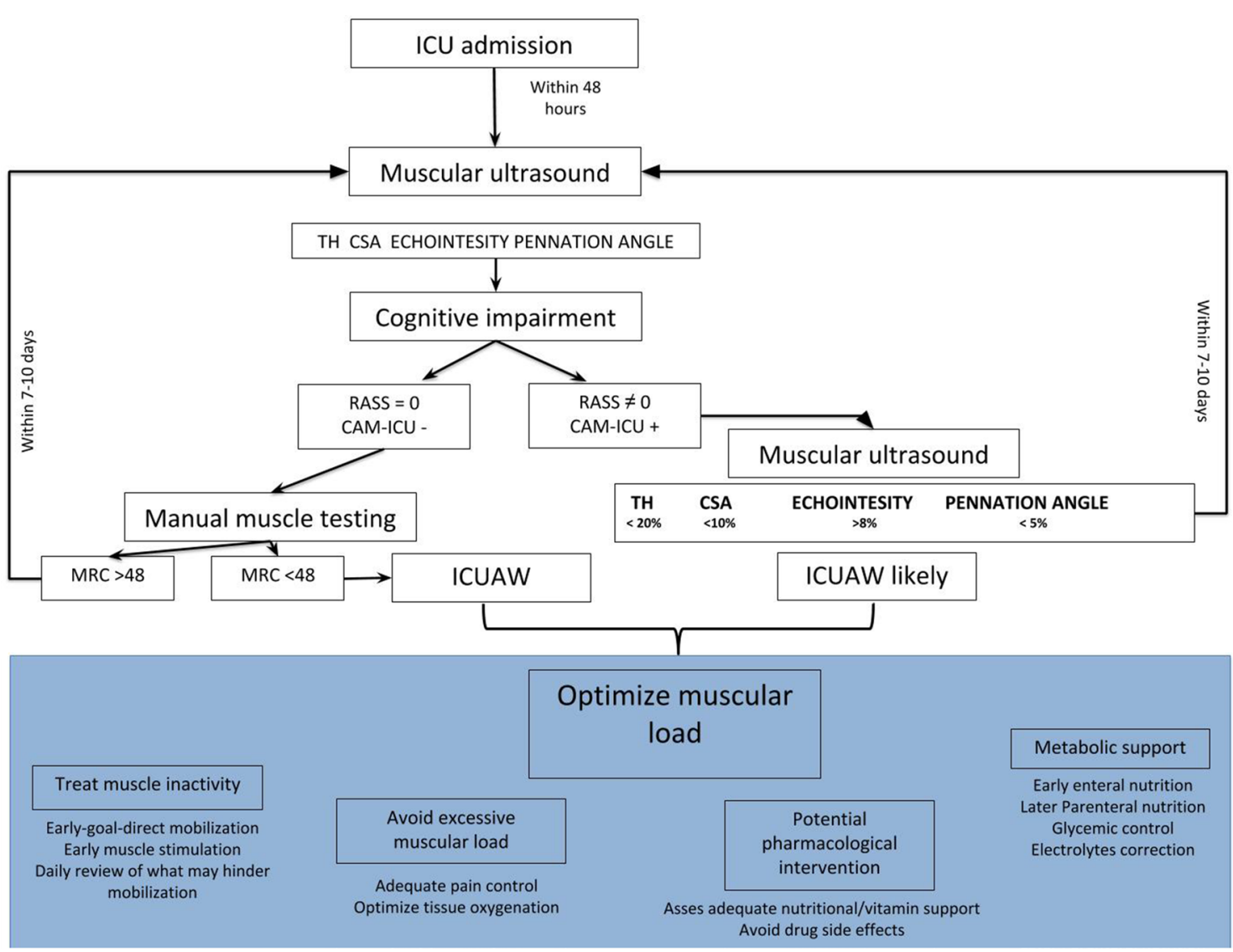

Fig. 6 The muscle ultrasound flowchart for the assessment and minimization of ICUAW. This flowchart suggests a protocol for logical and early identification of ICUAW. Ideally, within the first $48 \mathrm{~h}$, a first muscle ultrasound assessment should be performed for a baseline picture of patient muscle characteristics (the evaluation should at least regard the quadriceps rectus femoris, and it may be "omni-comprehensive" of muscle thickness (TH), cross-sectional area (CSA), echointensity (if the operator is familiar with any image editing software), pennation angle. At the same time, the cognitive impairment should be evaluated using standard reproducible scales (such as the Richmond agitation sedation scale and the confusion assessment method for the ICU). If these scores are in the normal range, the application of manual muscle testing such as the medical research council scale is possible. These first evaluations might be reconsidered within the first 7-10 days after the admission in the ICU, and their modifications over time, integrated with each other as well as with the reevaluation of MRC scale, allow an accurate diagnosis of ICUAW and should be used to modify the different patient-dependent factors, such as pharmacological strategies, muscular overloading or inactivity, and metabolic derangements. RASS Richmond agitation sedation scale, CAM-ICU confusion assessment method for the ICU, ICU intensive care unit, MRC Medical Research Council scale, TH muscle thickness, CSA cross-sectional area, ICUAW ICU-acquired weakness 
for ICUAW, with the aim of optimizing muscle load; in this case, serial reevaluations by muscular ultrasound may represent valuable tools. In this regard, reductions of $20 \%$ in muscle thickness, $10 \%$ of CSA, $5 \%$ of pennation angle and an increment in echointensity of at least $8 \%$ [66] seem reasonable indicators of ICUAW, even if the latter technique has not been standardized and no clear cutoff has yet been determined [72].

\section{Conclusions}

Skeletal muscle wasting in the critically ill has significant functional implications for patients who survive, and the development of prophylactic or therapeutic interventions has been troubled by our lack of understanding of the pathophysiology driving the process of muscle wasting. Several studies have demonstrated that muscle ultrasound is able to reliably detect pathological changes, especially once it is performed repeatedly. Muscle ultrasound might help to identify those patients at highest risk of prolonged complications, which result from excess muscle catabolism. Despite this intriguing potential, the interpretation of the available studies is difficult because of significant methodological defects, inadequate sample sizes, and lack of standardization of the ultrasound methodology. Nevertheless, further studies are certainly needed to describe the detailed time course of ultrasonic muscle changes and the progression of spontaneous activity, particularly in relation to the functional clinical outcome.

\section{Abbreviations \\ ICU: intensive care unit; NMBAs: neuromuscular blockade agents; ICUAW : intensive care unit-acquired weakness; CT: computed tomography; MRI: magnetic resonance imaging; DEXA: dual-energy X-ray absorptiometry; CSA: cross-sectional area; TH: muscles layer thickness; FL: fascicle length; LOS: length of stay; FFM: free fatty mass; RF: rectus femoris; MRCs: Medical Research Council scale; RASS: Richmond agitation sedation scale; CAM: confusion assessment method.}

\section{Acknowledgements}

We are grateful to Prof. John J. Marini for his precious help in editing English revision.

\section{Authors' contributions}

PF performed the main literature research and was a major contributor in writing the manuscript; MU and DC reviewed the manuscript and the table; SC and SF completed the figures editing process; all authors read and approved the final manuscript.

\section{Funding}

No funding for this review.

\section{Availability of data and materials} Not applicable.

Ethics approval and consent to participate Not applicable.
Consent for publication

Not applicable.

\section{Competing interests}

The authors declare that they have no competing interests.

\section{Author details}

${ }^{1}$ SC Anestesia e Rianimazione, Ospedale San Paolo - Polo Universitario, ASST Santi Paolo e Carlo, Milan, Italy. ${ }^{2}$ Dipartimento di Scienze della Salute, Università degli Studi di Milano, Milan, Italy. ${ }^{3}$ Centro Ricerca Coordinata di Insufficienza Respiratoria, Università degli Studi di Milano, Milan, Italy.

Received: 10 December 2018 Accepted: 10 May 2019

Published online: 17 May 2019

\section{References}

1. Kress JP, Hall JB. ICU-acquired weakness and recovery from critical illness. N Engl J Med. 2014;370:1626-35.

2. Hermans $G$, Van den Berghe $G$. Clinical review: intensive care unit acquired weakness. Crit Care. 2015;19:274.

3. Cheung AM, Tansey CM, Tomlinson G, Diaz-Granados N, Matté A, Barr A, et al. Two-year outcomes, health care use, and costs of survivors of acute respiratory distress syndrome. Am J Respir Crit Care Med. 2006:174:538-44

4. Cuthbertson BH, Roughton S, Jenkinson D, Maclennan G, Vale L. Quality of life in the five years after intensive care: a cohort study. Crit Care. 2010;14:R6.

5. Herridge MS, Cheung AM, Tansey CM, Matte-Martyn A, Diaz-Granados N, Al-Saidi F, et al. One-year outcomes in survivors of the acute respiratory distress syndrome. N Engl J Med. 2003;348:683-93.

6. Myhren $H$, Ekeberg $\varnothing$, Stokland $O$. Health-related quality of life and return to work after critical illness in general intensive care unit patients: a 1-year follow-up study. Crit Care Med. 2010;38:1554-61.

7. Bednarík J, Vondracek P, Dusek L, Moravcova E, Cundrle I. Risk factors for critical illness polyneuromyopathy. J Neurol. 2005;252:343-51.

8. Bednarik J, Lukas Z, Vondracek P. Critical illness polyneuromyopathy: the electrophysiological components of a complex entity. Intensive Care Med. 2003;29:1505-14

9. Allen DC, Arunachalam R, Mills KR. Critical illness myopathy: further evidence from muscle-fiber excitability studies of an acquired channelopathy. Muscle Nerve. 2008;37:14-22.

10. Novak KR, Nardelli P, Cope TC, Filatov G, Glass JD, Khan J, et al. Inactivation of sodium channels underlies reversible neuropathy during critical illness in rats. J Clin Invest. 2009;119:1150-8.

11. Teener JW, Rich MM. Dysregulation of sodium channel gating in critical illness myopathy. J Muscle Res Cell Motil. 2006;27:291-6.

12. Zhou C, Wu L, Ni F, Ji W, Wu J, Zhang H. Critical illness polyneuropathy and myopathy: a systematic review. Neural Regen Res. 2014;9:101-10.

13. Latronico N, Herridge M, Hopkins RO, Angus D, Hart N, Hermans G, et al. The ICM research agenda on intensive care unit-acquired weakness. Intensive Care Med. 2017:43:1270-81.

14. Moisey LL, Mourtzakis M, Cotton BA, Premji T, Heyland DK, Wade CE, et al. Skeletal muscle predicts ventilator-free days, ICU-free days, and mortality in elderly ICU patients. Crit Care. 2013;17:R206.

15. De Jonghe B, Bastuji-Garin S, Sharshar T, Outin H, Brochard L. Does ICU-acquired paresis lengthen weaning from mechanical ventilation? Intensive Care Med. 2004;30:1117-21.

16. Puthucheary ZA, Rawal J, McPhail M, Connolly B, Ratnayake G, Chan $P$, et al. Acute skeletal muscle wasting in critical illness. JAMA. 2013:310:1591-600.

17. Arts IMP, Pillen S, Schelhaas HJ, Overeem S, Zwarts MJ. Normal values for quantitative muscle ultrasonography in adults. Muscle Nerve. 2010;41:32-41.

18. Batt J, dos Santos CC, Cameron Jl, Herridge MS. Intensive care unitacquired weakness: clinical phenotypes and molecular mechanisms. Am J Respir Crit Care Med. 2013;187:238-46.

19. Babb T, Levine B, Philley J. ICU-acquired weakness: an extension of the effects of bed rest. Am J Respir Crit Care Med. 2012;185:230-1. 
20. Nanas S, Kritikos K, Angelopoulos E, Siafaka A, Tsikriki S, Poriazi M, et al. Predisposing factors for critical illness polyneuromyopathy in a multidisciplinary intensive care unit. Acta Neurol Scand. 2008;118:175-81.

21. Bittner EA, Martyn JA, George E, Frontera WR, Eikermann M. Measurement of muscle strength in the intensive care unit. Crit Care Med. 2009;37:S321-30.

22. Waak K, Zaremba S, Eikermann M. Muscle strength measurement in the intensive care unit: not everything that can be counted counts. J Crit Care. 2013:28:96-8.

23. Denehy L, de Morton NA, Skinner EH, Edbrooke L, Haines K, Warrillow S, et al. A physical function test for use in the intensive care unit: validity, responsiveness, and predictive utility of the physical function ICU test (scored). Phys Ther. 2013;93:1636-45.

24. Reeves ND, Maganaris CN, Narici MV. Ultrasonographic assessment of human skeletal muscle size. Eur J Appl Physiol. 2004;91:116-8.

25. Dupont AC, Sauerbrei EE, Fenton PV, Shragge PC, Loeb GE, Richmond FJ. Real-time sonography to estimate muscle thickness: comparison with MRI and CT. J Clin Ultrasound. 2001;29:230-6.

26. Miyatani M, Kanehisa H, Kuno S, Nishijima T, Fukunaga T. Validity of ultrasonograph muscle thickness measurements for estimating muscle volume of knee extensors in humans. Eur J Appl Physiol. 2002;86:203-8.

27. Connolly BA, Jones GD, Curtis AA, Murphy PB, Douiri A, Hopkinson NS, et al. Clinical predictive value of manual muscle strength testing during critical illness: an observational cohort study. Crit Care. 2013;17:R229.

28. Paris MT, Mourtzakis M, Day A, Leung R, Watharkar S, Kozar R, et al. Validation of bedside ultrasound of muscle layer thickness of the quadriceps in the critically ill patient (VALIDUM Study). JPEN J Parenter Enteral Nutr. 2017;41:171-80

29. Connolly B, MacBean V, Crowley C, Lunt A, Moxham J, Rafferty GF, et al. Ultrasound for the assessment of peripheral skeletal muscle architecture in critical illness: a systematic review. Crit Care Med. 2015;43:897-905.

30. Bunnell A, Ney J, Gellhorn A, Hough CL. Quantitative neuromuscular ultrasound in intensive care unit-acquired weakness: a systematic review. Muscle Nerve. 2015;52:701-8.

31. Hadda V, Kumar R, Hussain T, Khan MA, Madan K, Mohan A, et al. Reliability of ultrasonographic arm muscle thickness measurement by various levels of health care providers in ICU. Clin Nutr ESPEN. 2018;24:78-81.

32. Turton P, Hay R, Taylor J, McPhee J, Welters I. Human limb skeletal muscle wasting and architectural remodeling during five to ten days intubation and ventilation in critical care-an observational study using ultrasound. BMC Anesthesiol. 2016;16:119.

33. Mueller N, Murthy S, Tainter CR, Lee J, Riddell K, Fintelmann FJ, et al. Can sarcopenia quantified by ultrasound of the rectus femoris muscle predict adverse outcome of surgical intensive care unit patients as well as frailty? A prospective, observational cohort study. Ann Surg. 2016;264:1116-24.

34. Annetta MG, Pittiruti M, Silvestri D, Grieco DL, Maccaglia A, La Torre MF, et al. Ultrasound assessment of rectus femoris and anterior tibialis muscles in young trauma patients. Ann Intensive Care. 2017;7:104

35. Palakshappa JA, Reilly JP, Schweickert WD, Anderson BJ, Khoury V, Shashaty MG, et al. Quantitative peripheral muscle ultrasound in sepsis: muscle area superior to thickness. J Crit Care. 2018;47:324-30.

36. Peetrons P. Ultrasound of muscles. Eur Radiol. 2002;12:35-43.

37. Pillen S, van Keimpema M, Nievelstein RAJ, Verrips A, van KruijsbergenRaijmann W, Zwarts MJ. Skeletal muscle ultrasonography: visual versus quantitative evaluation. Ultrasound Med Biol. 2006:32:1315-21.

38. Strasser EM, Draskovits T, Praschak M, Quittan M, Graf A. Association between ultrasound measurements of muscle thickness, pennation angle, echogenicity and skeletal muscle strength in the elderly. Age (Dordr). 2013;35:2377-88.

39. Schedel H, Reimers CD, Nägele M, Witt TN, Pongratz DE, Vogl T. Imaging techniques in myotonic dystrophy. A comparative study of ultrasound, computed tomography and magnetic resonance imaging of skeletal muscles. Eur J Radiol. 1992;15:230-8.

40. Mercuri E, Pichiecchio A, Allsop J, Messina S, Pane M, Muntoni F. Muscle MRI in inherited neuromuscular disorders: past, present, and future. J Magn Reson Imaging. 2007;25:433-40.

41. Shepherd S, Batra A, Lerner DP. Review of critical illness myopathy and neuropathy. Neurohospitalist. 2017;7:41-8.

42. Lacomis D. Electrophysiology of neuromuscular disorders in critical illness. Muscle Nerve. 2013;47:452-63.
43. Hough CL, Lieu BK, Caldwell ES. Manual muscle strength testing of critically ill patients: feasibility and interobserver agreement. Crit Care. 2011;15:R43.

44. Witteveen E, Sommers J, Wieske L, Doorduin J, van Alfen N, Schultz MJ, et al. Diagnostic accuracy of quantitative neuromuscular ultrasound for the diagnosis of intensive care unit-acquired weakness: a cross-sectional observational study. Ann Intensive Care. 2017;7:40.

45. Akima H, Kubo K, Imai M, Kanehisa H, Suzuki Y, Gunji A, et al. Inactivity and muscle: effect of resistance training during bed rest on muscle size in the lower limb. Acta Physiol Scand. 2001;172:269-78.

46. Ikai M, Fukunaga T. Calculation of muscle strength per unit cross-sectional area of human muscle by means of ultrasonic measurement. Int Z Angew Physiol. 1968;26:26-32.

47. Cagnie B, Derese E, Vandamme L, Verstraete K, Cambier D, Danneels L. Validity and reliability of ultrasonography for the longus colli in asymptomatic subjects. Man Ther. 2009;14:421-6.

48. Cartwright MS, Demar S, Griffin LP, Balakrishnan N, Harris JM, Walker FO. Validity and reliability of nerve and muscle ultrasound. Muscle Nerve. 2013:47:515-21.

49. Blazevich AJ, Gill ND, Zhou S. Intra- and intermuscular variation in human quadriceps femoris architecture assessed in vivo. J Anat. 2006;209:289-310.

50. Thoirs K, English C. Ultrasound measures of muscle thickness: intra-examiner reliability and influence of body position. Clin Physiol Funct Imaging. 2009;29:440-6.

51. Abe T, Loenneke JP, Thiebaud RS. Morphological and functional relationships with ultrasound measured muscle thickness of the lower extremity: a brief review. Ultrasound. 2015;23:166-73.

52. Gruther W, Benesch T, Zorn C, Paternostro-Sluga T, Quittan M, FialkaMoser $V$, et al. Muscle wasting in intensive care patients: ultrasound observation of the M. quadriceps femoris muscle layer. J Rehabil Med. 2008:40:185-9.

53. Reimers K, Reimers CD, Wagner S, Paetzke I, Pongratz DE. Skeletal muscle sonography: a correlative study of echogenicity and morphology. J Ultrasound Med. 1993;12:73-7.

54. Pillen S, van Dijk JP, Weijers G, Raijmann W, de Korte CL, Zwarts MJ. Quantitative gray-scale analysis in skeletal muscle ultrasound: a comparison study of two ultrasound devices. Muscle Nerve. 2009;39:781-6.

55. Heckmatt JZ, Leeman S, Dubowitz V. Ultrasound imaging in the diagnosis of muscle disease. J Pediatr. 1982;101:656-60.

56. Fukunaga T, Kawakami Y, Kuno S, Funato K, Fukashiro S. Muscle architecture and function in humans. J Biomech. 1997;30:457-63.

57. Rutherford OM, Jones DA. Measurement of fibre pennation using ultrasound in the human quadriceps in vivo. Eur J Appl Physiol Occup Physiol. 1992;65:433-7

58. Sarwal A, Parry SM, Berry MJ, Hsu F-C, Lewis MT, Justus NW, et al. Interobserver reliability of quantitative muscle sonographic analysis in the critically ill population. J Ultrasound Med. 2015;34:1191-200.

59. Sharshar T, Bastuji-Garin S, Stevens RD, Durand M-C, Malissin I, Rodriguez $\mathrm{P}$, et al. Presence and severity of intensive care unit-acquired paresis at time of awakening are associated with increased intensive care unit and hospital mortality. Crit Care Med. 2009;37:3047-53.

60. De Jonghe B, Sharshar T, Lefaucheur J-P, Authier F-J, Durand-Zaleski I, Boussarsar $M$, et al. Paresis acquired in the intensive care unit: a prospective multicenter study. JAMA. 2002;288:2859-67.

61. Ali NA, O'Brien JM, Hoffmann SP, Phillips G, Garland A, Finley JCW, et al. Acquired weakness, handgrip strength, and mortality in critically ill patients. Am J Respir Crit Care Med. 2008;178:261-8.

62. Reid CL, Campbell IT, Little RA. Muscle wasting and energy balance in critical illness. Clin Nutr. 2004;23:273-80.

63. Freilich RJ, Kirsner RL, Byrne E. Isometric strength and thickness relationships in human quadriceps muscle. Neuromuscul Disord. 1995:5:415-22.

64. Baldwin CE, Paratz JD, Bersten AD. Diaphragm and peripheral muscle thickness on ultrasound: intra-rater reliability and variability of a methodology using non-standard recumbent positions. Respirology. 2011;16:1136-43.

65. Baldwin CE, Bersten AD. Alterations in respiratory and limb muscle strength and size in patients with sepsis who are mechanically ventilated. Phys Ther. 2014;94:68-82 
66. Grimm A, Teschner U, Porzelius C, Ludewig K, Zielske J, Witte OW, et al Muscle ultrasound for early assessment of critical illness neuromyopathy in severe sepsis. Crit Care. 2013;17:R227.

67. Jolley SE, Bunnell AE, Hough CL. ICU-acquired weakness. Chest. 2016;150:1129-40.

68. Baldwin MR, Reid MC, Westlake AA, Rowe JW, Granieri EC, Wunsch H, et al. The feasibility of measuring frailty to predict disability and mortality in older medical-ICU survivors. J Crit Care. 2014;29:401-8.

69. Le Maguet P, Roquilly A, Lasocki S, Asehnoune K, Carise E, Saint Martin M, et al. Prevalence and impact of frailty on mortality in elderly ICU patients: a prospective, multicenter, observational study. Intensive Care Med. 2014;40:674-82

70. Cartwright MS, Kwayisi G, Griffin LP, Sarwal A, Walker FO, Harris JM, et al. Quantitative neuromuscular ultrasound in the intensive care unit. Muscle Nerve. 2013;47:255-9.

71. Pillen S, Arts IMP, Zwarts MJ. Muscle ultrasound in neuromuscular disorders. Muscle Nerve. 2008;37:679-93.

72. Parry SM, El-Ansary D, Cartwright MS, Sarwal A, Berney S, Koopman R, et al. Ultrasonography in the intensive care setting can be used to detect changes in the quality and quantity of muscle and is related to muscle strength and function. J Crit Care. 2015;30(1151):e9-14.

73. Greening NJ, Harvey-Dunstan TC, Chaplin EJ, Vincent EE, Morgan MD, Singh SJ, et al. Bedside assessment of quadriceps muscle by ultrasound after admission for acute exacerbations of chronic respiratory disease. Am J Respir Crit Care Med. 2015;192:810-6.
74. Segaran E, Wandrag L, Stotz M, Terblanche M, Hickson M. Does body mass index impact on muscle wasting and recovery following critical illness? A pilot feasibility observational study. J Hum Nutr Diet. 2017;30:227-35.

75. Puthucheary ZA, McNelly AS, Rawal J, Connolly B, Sidhu PS, Rowlerson $A$, et al. Rectus femoris cross-sectional area and muscle layer thickness: comparative markers of muscle wasting and weakness. Am J Respir Crit Care Med. 2017;195:136-8.

76. Ely EW, Truman B, Shintani A, Thomason JWW, Wheeler AP, Gordon S, et al. Monitoring sedation status over time in ICU patients: reliability and validity of the Richmond Agitation-Sedation Scale (RASS). JAMA. 2003;289:2983-91.

77. Gusmao-Flores D, Salluh JIF, Chalhub RÁ, Quarantini LC. The confusion assessment method for the intensive care unit (CAM-ICU) and intensive care delirium screening checklist (ICDSC) for the diagnosis of delirium: a systematic review and meta-analysis of clinical studies. Crit Care. 2012;16:R115

\section{Publisher's Note}

Springer Nature remains neutral with regard to jurisdictional claims in published maps and institutional affiliations.

\section{Submit your manuscript to a SpringerOpen ${ }^{\circ}$ journal and benefit from:}

- Convenient online submission

- Rigorous peer review

- Open access: articles freely available online

- High visibility within the field

- Retaining the copyright to your article

Submit your next manuscript at $\boldsymbol{\nabla}$ springeropen.com 